Meta

Journal des traducteurs

Translators' Journal

\title{
La traduction à vue en interprétation simultanée : quelle opérationnalité ambitionner?
}

\section{Frans De Laet et Raymond Vanden Plas}

Volume 50, numéro 4, décembre 2005

Pour une traductologie proactive - Actes

For a Proactive Translatology - Proceedings

Por una traductología proactiva - Actas

URI : https://id.erudit.org/iderudit/019835ar

DOI : https://doi.org/10.7202/019835ar

Aller au sommaire du numéro

Éditeur(s)

Les Presses de l'Université de Montréal

ISSN

0026-0452 (imprimé)

1492-1421 (numérique)

Découvrir la revue

Citer cet article

De Laet, F. \& Vanden Plas, R. (2005). La traduction à vue en interprétation simultanée : quelle opérationnalité ambitionner ? Meta, 50(4).

https://doi.org/10.7202/019835ar
Résumé de l'article

Quiconque parcourt la mission de l'interprète lors de réunions institutionnelles des dernières décennies confirmera que les conditions de travail ont subi une véritable métamorphose. Si la durée des assemblées est restée stable, l'ampleur croissante des ordres du jour, par contre, ne peut être niée. Il en résulte que l'intervenant d'aujourd'hui se trouve confronté à un temps de parole limité. Il tient, néanmoins, à la totalité de son discours et pratique, à cette fin, la lecture accélérée de son texte.

Heureusement (?), l'interprète reçoit le texte, souvent peu avant le début de la réunion, parfois même quand il est déjà en cabine. Comment doit-il, peut-il se servir du document ? Pour le formateur se pose la question de la préparation de l'étudiant à ce type de travail. Notre communication porte sur l'expérience avec un groupe d'étudiants en dernière année de formation. L'objectif principal : examiner jusqu'à quel moment, l'opérationnalité de la traduction à vue en interprétation simultanée est performante. 


\title{
La traduction à vue en interprétation simultanée : quelle opérationnalité ambitionner?
}

\author{
FRANS DE LAET \\ Haute École de Bruxelles, Bruxelles, Belgique \\ fdelaet@heb.be \\ RAYMOND VANDEN PLAS \\ Haute École de Bruxelles, Bruxelles, Belgique \\ rvandenplas@heb.be
}

\begin{abstract}
RÉSUMÉ
Quiconque parcourt la mission de l'interprète lors de réunions institutionnelles des dernières décennies confirmera que les conditions de travail ont subi une véritable métamorphose. Si la durée des assemblées est restée stable, l'ampleur croissante des ordres du jour, par contre, ne peut être niée. Il en résulte que l'intervenant d'aujourd'hui se trouve confronté à un temps de parole limité. Il tient, néanmoins, à la totalité de son discours et pratique, à cette fin, la lecture accélérée de son texte.

Heureusement (?), l'interprète reçoit le texte, souvent peu avant le début de la réunion, parfois même quand il est déjà en cabine. Comment doit-il, peut-il se servir du document ? Pour le formateur se pose la question de la préparation de l'étudiant à ce type de travail. Notre communication porte sur l'expérience avec un groupe d'étudiants en dernière année de formation. L'objectif principal : examiner jusqu'à quel moment, l'opérationnalité de la traduction à vue en interprétation simultanée est performante.
\end{abstract}

\begin{abstract}
Anyone who has taken on interpreting within the framework of institutional meetings over the past decades is bound to confirm that working conditions have changed quite dramatically. Where the length of meetings has remained by and large stable, the sheer extent of the agendas has, by contrast, rocketed. As a result, today's participants taking the floor have to stick to limited speaking times and if they wish to deliver their entire speech they have no other choice but to speed up their reading pace.

Fortunately (?), the interpreters often receive their texts shortly before the meetings begin, but there are times when they only receive them once they are in their booths. How should they / how can they use this material? Hence the onus on the trainers to groom their students in this respect. Our paper focuses on an experiment carried out with a group of students in their final year. The main aim is to see until when exactly sight translation in simultaneous interpreting is operational.
\end{abstract}

\section{MOTS-CLÉS/KEYWORDS}

interprétation simultanée, traduction à vue, formation, operationnalité

L'interprète de conférence est aujourd'hui confronté à un défi de plus en plus récurrent :

l'interprétation de textes lus. Le phénomène n'est certes pas nouveau mais il est de plus en plus fréquent, il risque de devenir demain le lot quotidien de l'interprète. Ceci concerne aussi bien le marché libre que le marché des Institutions Internationales.

L'évolution est impressionnante. Il y a une trentaine d'années, les règles de l'AIIC étaient incontournables, il était interdit de lire un texte en réunion. Quiconque souhaitait prononcer une allocution devait la faire parvenir aux interprètes plusieurs jours avant la réunion. Si quelqu'un lisait un texte dont les interprètes ne disposaient pas, ceux-ci arrêtaient de travailler et la réunion était suspendue pour qu'ils puissent prendre connaissance du document. Nous sommes loin du compte 
aujourd'hui. De plus en plus fréquemment, les interprètes sont confrontés à des textes lus dont ils n'ont pas de copie ou qui leur sont remis quelques instants avant la présentation.

La première question qui vient à l'esprit est de savoir comment cette évolution a-t-elle pu se produire et pourquoi les interprètes ne s'y sont pas opposés. Les causes de l'évolution sont évidentes. Nous vivons dans un monde où la pression du temps est un facteur permanent, les temps de parole sont limités et la lecture rapide de plus en plus fréquente. Prenons l'exemple des Institutions Européennes. Dans les années soixante-dix personne ne lisait, tout le monde parlait librement, le travail de l'interprète était alors passionnant et valorisant. Aujourd'hui la lecture est monnaie courante, en cause les élargissements de l'Union Européenne. Lorsque 1'Union comptait six États membres, une réunion ministérielle durait un jour ou un jour et demi. Aujourd'hui, l'Union Européenne compte 25 États membres et les réunions ministérielles durent un jour ou un jour et demi. Chaque ministre se voit donc imposer un temps de parole très strict et il est plus facile de faire passer le maximum d'arguments en focalisant toute son attention sur un écrit préparé à l'avance qu'en réfléchissant à haute voix.

En réalité, ce qui intéresse les intervenants, c'est que leurs propos soient enregistrés et figurent aux actes de la réunion, peu importe la forme de la communication. Ceci ne facilite bien sûr pas la tâche de l'interprète mais surtout cela entraine une baisse de la qualité des réunions. Van Hoof Haferkamp, ancien directeur général du SCIC (Service commun Interprétation-conférences), faisait remarquer à juste titre que dès que quelqu'un lit, plus personne n'écoute. Il y a aujourd'hui de moins en moins d'écoute et donc de moins en moins de débats. Les vraies négociations ont lieu dans les couloirs. Tout ceci rend le travail de l'interprète particulièrement frustrant. L'interprétation devient un exercice de haute voltige, sans filet. L'interprète est appélé à faire un travail difficile, épuisant, dont le résultat est rarement parfait. En outre, il constate que les participants ne l'écoutent souvent que d'une oreille.

Pourquoi les interprètes ne se sont-ils pas opposés à cette évolution ? Tout simplement, parce qu'ils n'en ont pas eu les moyens. L'interprétation est un service : si le preneur de service se contente d'une qualité moindre parce qu'il impose au prestataire des conditions de travail pénibles, il est difficile de refuser de s'y plier. Chacun dans la profession, des formateurs aux responsables des grands services d'interprétation des Institutions Internationales, en passant bien sûr par les « interprètes de terrain », a pris conscience du phénomène et s'efforce d'y apporter remède.

En matière de formation, beaucoup estiment qu'il faudrait mettre davantage l'accent sur la traduction à vue afin de tenir compte de cette évolution. En réalité, la traduction à vue constitue déjà un outil pédagogique utile pour l'enseignement de la technique d'interprétation simultanée traditionnelle (c'est-à-dire une réunion où les discours ne sont pas lus). La traduction à vue permet à l'étudiant d'acquérir une gymnastique de l'esprit, de capter rapidement le message, de le transposer tout en prenant déjà connaissance visuellement de l'idée suivante, de perfectionner la souplesse de la langue d'arrivée et souvent d'améliorer sa connaissance de la langue passive (Seleskovitch-Lederer 2002 : 204-208). Beaucoup de formateurs considèrent qu'être capable de faire une bonne traduction à vue, en ne collant pas au texte original et en transmettant le message dans une langue élégante est le signe d'un véritable potentiel pour la simultanée.

La preuve en est que dans beaucoup d'écoles d'interprètes, les candidats sont soumis à un test de traduction à vue lors des épreuves d'admission ou d'orientation.

À l'université d'Ottawa e.a., la « sight interpretation » fait partie des épreuves d'admission afin de vérifier les réflexes d'un candidat par rapport à un sujet qu'il ne peut pas encore interpréter (Lambert 1991). Les interprètes qui présentent le test de recrutement de l'OTAN se voient également imposer une épreuve de traduction à vue alors que les interprètes qui travaillent pour l'OTAN ne sont jamais appelés à ce type de mission.

On a tendance à croire que l'interprétation simultanée à partir d'un texte facilite la transition entre la traduction à vue et l'interprétation simultanée traditionnelle (discours non lus). Nous nous inscrivons en faux contre cette affirmation, ce serait mettre la charrue avant les bœufs. L'apprentissage de l'interprétation simultanée ne peut se faire sur la base de textes lus, elle doit se faire sur la base de discours prononcés librement, à un rythme raisonnable, pour que l'étudiant puisse apprendre à écouter, à percevoir le message et à le transmettre. Commencer l'enseignement de la simultanée par des exposés lus, fera prendre à l'interprète la très mauvaise habitude de coller à 
l'orateur, de traduire des mots et pas des idées et pourquoi ne pas le dire, de le décourager rapidement et définitivement face à la difficulté de la tâche. Commencer l'enseignement de la simultanée par des conférences lues équivaut à de l'assassinat pédagogique. Le jeune interprète doit se sentir à l'aise dans sa cabine, il doit apprendre à gérer le stress de l'interprétation simultanée « normale », à maîtriser la technique de l'écoute et de la transmission en sachant que des éléments tels que le langage corporel de l'orateur et les réactions de son audience sont des paramètres visibles non négligeables. Ce n'est qu'après avoir acquis cette technique qu'il pourra aborder avec sérénité et chances de succès le raz de marée de stress qu'entraîne l'interprétation de communications lues rapidement. Nous reviendrons plus avant sur l'utilisation de la technique de traduction à vue en cabine dans les cas où l'interprète dispose du texte lu, sur la base d'une expérience réalisée à l'ISTI - Bruxelles.

Les Institutions Européennes ont elles aussi conscience de cette évolution peu valorisante. À la Commission européenne, la DG SCIC, a élaboré un dépliant à l'intention des orateurs «Vous prenez la parole : quelques conseils »; le troisième conseil «ne lisez pas, parlez » n'est malheureusement pas toujours respecté. En outre, sous la rubrique « réunions multilingues : mode d'emploi » figure une recommandation très claire :

«Si vous devez lire un texte préparé ou une déclaration, veuillez vous assurer que des exemplaires en ont été préalablement remis aux interprètes. Ce texte sera traité en toute confidentialité et seule la version prononcée fera foi » (http://europa.eu.int/comm/scic/help/newflyer_fr.pdf).

Le SCIC organise également des séances d'information à l'adresse des nouveaux fonctionnaires appelés à prendre la parole en réunion afin de les sensibiliser aux problèmes de l'interprétation.

Lors de la $9^{\mathrm{e}}$ Conférence DG SCIC-Universités sur le thème «EU 25 : Premier bilan » qui s'est tenue à Bruxelles le 04 mars 2005, Esteban Causo et Fox ont présenté un projet pilote, le Speech repository Study, consacré au développement d'un outil de formation électronique pour interprètes et étudiants en interprétation.

Une des possibilités offertes par ce programme est le défilement du texte sur un quart de l'écran (moniteur), ce qui permet à l'interprète d'utiliser la traduction à vue en tant que support de son interprétation simultanée, sans toutefois avoir la possibilité d'annoter.

Face à une intervention lue, il est essentiel pour l'interprète de choisir sa stratégie. Celle-ci sera fonction de toute une série d'éléments. Avoir le texte sous les yeux ne sera pas nécessairement garantie d'une prestation de qualité ou d'un travail aisé.

Nous avons réalisé une expérience avec un groupe de 10 étudiants en interprétation de dernière année à l'ISTI. Nous avons choisi des étudiants pour leur disponibilité et surtout parce qu'ils ne possèdent pas encore toutes les ficelles du métier pour cacher leurs difficultés.

Les étudiants étaient tous volontaires, ils étaient de langue maternelle française, italienne, espagnole, allemande, polonaise et slovène. La langue source fut principalement l'anglais, parfois le français. L'expérience a porté sur une période de huit semaines, à raison d'une demi journée par semaine. Elle s'est limitée à l'interprétation de textes lus dont les étudiants disposaient, sans quoi, nous aurions été hors sujet.

Notre objectif principal n'était pas d'étudier le partage d'attention entre deux activités ou d'essayer de savoir quel type d'activité marque le plus la mémoire de l'interprète (Viezzi 1989 \& 1990 - Lambert 2004), mais simplement d'observer la stratégie choisie par les étudiants et d'en évaluer l'opérationnalité.

Nous avons confronté les étudiants avec le plus grand nombre d'hypothèses :

- difficulté variable des textes : il s'agissait toujours de textes d'actualité (plénière du parlement européen, allocution de personnalités politiques à l'occasion de missions ou de rencontres importantes, commentaires sur des événements politiques, rapports de groupes d'études, etc.) mais le niveau de difficulté était très variable, du discours lu mais rédigé en style oral au rapport riche en informations, truffé de chiffres et de sigles ; 
- présentation différente des textes : en colonnes ou non, avec simple ou double interligne, en petits ou grands caractères ;

- respect ou non du texte écrit : au début de l'expérience, l'orateur a respecté scrupuleusement la version écrite. Au cours de l'expérience nous avons glissé des difficultés inattendues dans l'exercice : omission de paragraphes entiers, de parties de paragraphes, modifications de l'ordre d'une énumération, modifications de chiffres ;

- variation du rythme de la lecture : les étudiants ont été confrontés à des accélérations soudaines du débit, qui a varié de \pm 105 mots par minute (mode lecture croisière) à 145 mots par minute (mode lecture soutenue);

- variation de la durée des interventions : de \pm 5 minutes à plus de 20 minutes (le texte le plus long fut de 293 lignes / durée de la lecture : 21 minutes et 50 secondes);

- délais différents de réception du texte par les étudiants : document remis soit deux jours avant l'interprétation, soit la veille, soit 10 à 15 minutes avant la prestation ou au moment même du début de l'exercice.

Nous n'avions donné aux étudiants aucune instruction stricte. Chacun était libre de son mode de préparation et de la façon d'utiliser le texte. Mais nous les avons simplement mis en garde contre les télescopages (coller le début de la première idée à la fin de la deuxième). Il vaut en effet mieux avoir 50\% des idées plutôt que 50\% de chaque idée. Nous leur avons également demandé de ne pas traduire par écrit les textes reçus la veille ou deux jours avant la réunion. Aucun interprète professionnel n'envisagerait de faire deux fois le travail et en outre, seul le texte lu faisant foi, il est impensable pour l'interprète de lire un texte traduit. Finalement, les étudiants ont été informés des cas où ils seraient à deux par cabine. Ils ont pu alors se répartir le travail et à tout moment s'entraider (copilotage).

Afin de pouvoir se prononcer sur l'opérationnalité des prestations des étudiants, nous avons évoqué la méthode d'évaluation lors de tests d'interprétation au SCIC (Durand).

Quels sont les enseignements que nous avons pu tirer de cette expérience ?

1. la première constatation n'étonnera personne : les meilleures prestations - et de loin - ont été réalisées pour les textes distribués aux étudiants un ou deux jours à l'avance. Il est apparu qu'avec une bonne préparation ni la vitesse de lecture, ni la difficulté du discours ne constituait un obstacle à un travail de bonne qualité. Certes, toute modification apportée au document original constitue un élément déstabilisateur mais l'expérience a montré que les interprètes étaient en mesure de gérer cette difficulté.

Disposer du texte en temps utile :

- donne aux interprètes un sentiment de sécurité et de confiance (un facteur essentiel

d'une interprétation rassurante) ;

- $\quad$ permet de se familiariser avec le sujet ;

- facilite la recherche du vocabulaire spécifique et de la prononciation des noms propres ;

- $\quad$ permet le surlignement des chiffres ;

- $\quad$ aide à fixer les points de repérage ;

- $\quad$ contribue à l'élégance de la langue. 
2. pour les textes reçus 10 ou 15 minutes avant, l'interprète prend connaissance du contenu en lecture diagonale. La stratégie choisie fut de surligner les chiffres, les noms propres, les sigles, à l'aide de marqueurs de couleurs différentes. Le stress est, bien sûr, énorme, les étudiants ignorent la durée exacte de préparation, tout comme en situation réelle où l'interprète qui reçoit un document en réunion ignore souvent à quel moment l'orateur s'en servira. Il est clair que la préparation est incomplète et le résultat parfois décevant, mais cela reste préférable à une interprétation sans support écrit ou à une interprétation d'un texte remis au dernier moment.

3. lorsque le texte est remis au dernier moment, on doit constater qu'essayer de lire, tout en écoutant et en interprétant, est contre-productif, voire suicidaire, pour peu que l'orateur s'écarte de son original. Lors de la première session de l'expérience, tous les étudiants ont essayé de réaliser le triple exercice : écouter, suivre le texte « inconnu » et interpréter. Le résultat fut catastrophique :

- $\quad$ télescopage (début de la première idée collé à la fin de la suivante) ;

- $\quad$ langue maternelle négligée, voire incorrecte ;

- $\quad$ erreurs grammaticales ;

- $\quad$ voix chevrotante ;

- $\quad$ multiplication des interjections (« heu » etc.) ;

- $\quad$ contresens et non-sens, voire même désespoir (« mais je raconte n’importe quoi, moi ! »);

- $\quad$ décrochage complet.

Dès la deuxième session, les étudiants ont décidé de ne pas utiliser le texte, avec un résultat nettement plus satisfaisant. La seule exploitation utile du document relève de l'assistance du collègue (copilotage) lorsque les étudiants sont à deux en cabine ; le collègue peut suivre et surligner les chiffres, les noms propres, les sigles etc. Une excellente maîtrise des techniques de la traduction à vue n'élimine donc pas les difficultés lorsque l'orateur s'éloigne du texte et surtout lorsque le rythme s'accélère. Rien d'étonnant, notre champ de perception visuelle est limité et l'interprète perd pied quand sa capacité de rétention mémorielle est dépassée. Les problèmes constatés sont alors identiques à ceux rencontrés par les étudiants essayant d'utiliser l'écrit « inconnu ». En outre, la fatigue survient assez rapidement. Toutefois, il est apparu que ceux, qui possèdent cet affichage mnésique, grâce à la pratique régulière de la traduction à vue, résistent plus longtemps et réussissent avec un certain bonheur, l'interprétation de textes reçus au dernier moment, pourvu que le débit de lecture soit raisonnable et l'orateur ne s'éloigne pas (trop) de l'original. Ce qui hélas n'est jamais garanti.

4. En matière de préparation du texte les étudiants ont constaté que :

- $\quad$ la préparation individuelle est de loin préférable, chacun développant sa propre stratégie ;

- la configuration du texte est important : le double interligne, les grands caractères facilitent la préparation ;

- $\quad$ la durée moyenne de préparation pour les textes remis un ou deux jours à l'avance a diminué au fur et à mesure du déroulement de l'expérience : à titre d'illustration, il leur avait fallu 45 minutes de préparation pour le premier exercice dont la durée de lecture était de 15 minutes ;

- $\quad$ le brainstorming en groupe est une pratique qui nécessite un entraînement spécifique et un véritable esprit d'équipe. 


\section{Conclusions}

Si il y a quelques années, en vue de l'élargissement de l'Union Européenne, l'intraduisibilité en interprétation simultanée relevait plutôt du domaine de la faisabilité technique et technologique et de l'infrastructure - référons-nous au débat extraordinaire sur l'avenir de l'Union Européenne élargie qui s'est tenu à Strasbourg en novembre 2002 et lors duquel l'interprétation était assurée, pour la première fois, en 23 langues ; rappelons-nous également que dans le bâtiment Juste-Lipse du Conseil des ministres à Bruxelles, seules deux salles disposent de 20 cabines - aujourd'hui, l'intraduisibilité est plus souvent fonction de la présentation des interventions. De plus en plus, l'interprète se trouve confronté à des textes lus et souvent mal lus, il n'est pas rare que l'orateur prenne connaissance d'une déclaration préparée par un collaborateur en même temps que l'interprète.

Le premier conseil à donner aux étudiants-interprètes par leurs formateurs est de faire du discours leur propre discours, de se mettre dans la peau de l'orateur. Il est vrai que l'exercice devient ardu, si le discours n'est pas celui de l'orateur mais d'un tiers, s'il est mal lu et si, qui plus est, il est lu dans une langue qui n'est pas la langue maternelle de l'orateur. Que ce soit au sein des organisations internationales ou lors de congrès et séminaires privés, la couverture linguistique n'est presque jamais complète, les orateurs sont dès lors appelés à s'exprimer dans une langue autre que leur langue maternelle. Ce n'est pas souhaitable, mais incontournable.

Dans tous les cas, l'interprète (et l'étudiant-interprète) doit faire son choix : utiliser le document ou pas. Commencer sans texte et vouloir y retourner ensuite s'avère quasiment impossible, les points de repérage étant devenus introuvables. En outre, le consommateur aura la sensation malheureuse d'avoir à faire à une interprétation tantôt plus naturelle (travail de l'interprète sans texte ) tantôt plus artificielle (travail avec texte).

Finalement, nous ne pouvons que souscrire à l'impression que lire nuit de toute façon à la qualité de la compréhension aisée, de l'échange et du contact humain. Il sera peut-être difficile de changer la stratégie des orateurs qui sont tenus par des déclarations chronométrées et des impératifs de publication. Mais s'ils aspirent à une communication et une interprétation de haut niveau, ils devront à leur tour se mettre dans la peau de l'interprète. Car écouter, regarder, lire et «traduire » forment un ensemble qui rappelle l'image de la machine désassemblée, un statut que tout médiateur linguistique doit pouvoir décliner.

\section{RÉFÉRENCES}

DURAND, C. (2005): "Selection and Assessment of Interpreters", IX DG SCIC-Universities Conference "EU 25: A First Review”, Brussels, <http://europa.eu.int/comm/scic/interpreter/2005/progprev.pdf>.

Esteban Causo, J. and B. Fox (2005) : "Speech Repository Study", IX DG SCIC-Universities Conference "EU

25: A First Review”, Brussels, <http://europa.eu.int/comm/scic/interpreter/2005/progprev.pdf>.

LAMBERT, S. (1991) : «Aptitude Testing for Simultaneous Interpretation at the University of Ottawa", Meta 36-4 , p. 586-594.

LAMBERT, S. (2004): “Shared Attention during Sight Translation, Sight Interpretation and Simultaneous Interpretation", Meta 49-2, p. 294-306.

Seleskovitch, D. et M. LEDERER (2002): Pédagogie raisonnée de l'interprétation, Bruxelles, Didier Érudition Office des publications officielles des Communautés européennes.

VIEZZI, M. (1989): "Information Retention as a Parameter for the Comparison of Sight Translation and Simultaneous Interpretation: an Experimental Study”,, The Interpreters' Newsletter 2, p. 65-69.

VIEZZI, M. (1990): "Sight Translation, Simultaneous Interpretation and Information Retention", Laura Gran \& Christopher Taylor (eds.), Aspects of Applied and Experimental Research on Conference Interpretation", Udine, Campanotto, p. 54-60, <http://europa.eu.int/comm/scic/help/newflyer_fr.pdf>. 